\title{
UNIVERSITYOF BIRMINGHAM

\section{The relationship between obstructive sleep apnea and intra-epidermal nerve fiber density, PARP activation and foot ulceration in patients with type 2 diabetes}

\author{
Altaf, Quratul-ain Altaf; Ali, Asad; Piya, Milan K.; Raymond, Neil T.; Tahrani, Abd A.
}

DOI:

10.1016/j.jdiacomp.2016.05.025

License:

Creative Commons: Attribution-NonCommercial-NoDerivs (CC BY-NC-ND)

\section{Document Version}

Peer reviewed version

Citation for published version (Harvard):

Altaf, QA, Ali, A, Piya, MK, Raymond, NT \& Tahrani, AA 2016, 'The relationship between obstructive sleep apnea and intra-epidermal nerve fiber density, PARP activation and foot ulceration in patients with type 2 diabetes', Journal of Diabetes and its Complications, vol. 30, no. 7, pp. 1315-1320.

https://doi.org/10.1016/j.jdiacomp.2016.05.025

Link to publication on Research at Birmingham portal

\footnotetext{
General rights

Unless a licence is specified above, all rights (including copyright and moral rights) in this document are retained by the authors and/or the copyright holders. The express permission of the copyright holder must be obtained for any use of this material other than for purposes permitted by law.

- Users may freely distribute the URL that is used to identify this publication.

- Users may download and/or print one copy of the publication from the University of Birmingham research portal for the purpose of private study or non-commercial research.

- User may use extracts from the document in line with the concept of 'fair dealing' under the Copyright, Designs and Patents Act 1988 (?)

- Users may not further distribute the material nor use it for the purposes of commercial gain.

Where a licence is displayed above, please note the terms and conditions of the licence govern your use of this document.

When citing, please reference the published version.

Take down policy

While the University of Birmingham exercises care and attention in making items available there are rare occasions when an item has been uploaded in error or has been deemed to be commercially or otherwise sensitive.

If you believe that this is the case for this document, please contact UBIRA@lists.bham.ac.uk providing details and we will remove access to the work immediately and investigate.
} 


\section{Accepted Manuscript}

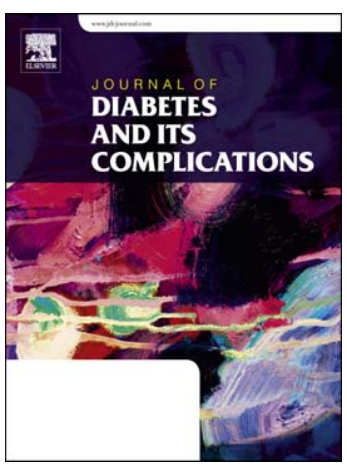

The relationship between obstructive sleep apnoea and intra epidermal nerve fiber density, PARP activation and foot ulceration in patients with type 2 diabetes

Q.A. Altaf, Asad Ali, Milan K. Piya, Neil T. Raymond, Abd A. Tahrani

PII:

S1056-8727(16)30179-9

DOI: doi: $10.1016 /$ j.jdiacomp.2016.05.025

Reference: JDC 6757

To appear in: $\quad$ Journal of Diabetes and Its Complications

Received date: 2 March 2016

Revised date: 3 May 2016

Accepted date: $\quad 28$ May 2016

Please cite this article as: Altaf, Q.A., Ali, A., Piya, M.K., Raymond, N.T. \& Tahrani, A.A., The relationship between obstructive sleep apnoea and intra epidermal nerve fiber density, PARP activation and foot ulceration in patients with type 2 diabetes, Journal of Diabetes and Its Complications (2016), doi: 10.1016/j.jdiacomp.2016.05.025

This is a PDF file of an unedited manuscript that has been accepted for publication. As a service to our customers we are providing this early version of the manuscript. The manuscript will undergo copyediting, typesetting, and review of the resulting proof before it is published in its final form. Please note that during the production process errors may be discovered which could affect the content, and all legal disclaimers that apply to the journal pertain. 


\section{The relationship between obstructive}

sleep apnoea and intra epidermal nerve fiber density, PARP activation and foot ulceration in patients with type 2 diabetes

Q A Altaf ${ }^{1,2}$, Asad Ali $^{3}$, Milan K Piya ${ }^{4,5}$, Neil T Raymond $^{6}$, Abd A Tahrani $^{1,2}$

${ }^{1}$ Department of Diabetes and Endocrinology, Heart of England NHS Foundation Trust, Birmingham, UK

${ }^{2}$ Institute of Metabolism and Systems, College of Medical and Dental Sciences, University of Birmingham, Birmingham, UK

${ }^{3}$ Department of Respiratory Medicine, University Hospitals Coventry and Warwickshire NHS Trust, Coventry, UK

${ }^{4}$ Warwickshire Institute for Diabetes, Endocrinology and Metabolism, University Hospitals Coventry and Warwickshire NHS Trust, Coventry, UK

${ }^{5}$ Institute of Digital Health, Warwick Manufacturing Group, University of Warwick, Coventry, UK

${ }^{6}$ Independent Epidemiology and Statistical Consultant, Epidemiology, Research Design and Statistical Consulting (ERDASC), Leicestershire, UK.

Corresponding author

Dr Abd A Tahrani

Department of Diabetes and Endocrinology,

Birmingham Heartlands Hospital, Birmingham B9 5SS, UK

Tel: $+44(0) 7801549960$

E mail: abd.tahrani@nhs.net

Word count 3103, Figures 3, Tables 2. 


\section{Abstract:}

Background: Obstructive sleep apnoea (OSA) is associated with increased nitrosative stress, endothelial dysfunction, and peripheral neuropathy in patients with type 2 diabetes. We hypothesized that OSA is associated with Poly ADP ribose polymerase (PARP) activation, lower intra epidermal nerve fibre density (IENFD), and diabetic foot ulceration (DFU).

Methods: A cross-sectional study of adults with type 2 diabetes recruited from a secondary care hospital in the UK. OSA was assessed by multi-channel home-based cardio-respiratory device (Alice PDX, Philips Respironics). DPN was assessed using the Michigan Neuropathy Screening Instrument (MNSI). IENFD and \% PAR stained nuclei were assessed using immunohistochemistry staining on skin biopsies. DFU was assessed based on MNSI.

Results: Skin biopsies and DFU data were available from 52 and 234 patients respectively. OSA was associated with lower IENFD (12.75 \pm 1.93 vs. $10.55 \pm 1.62$ vs. $9.42 \pm 1.16$ fibres $/ \mathrm{mm}$ of epidermis for no OSA, mild OSA and moderate to severe OSA respectively, $p<0.001$ ). Following adjustment, mild ( $B=-$ 2.19, $p=0.002)$ and moderate to severe OSA $(B=-3.45, p<0.001)$ were independently associated with IENFD. The apnoea hypopnea index (AHI) was associated with IENFD following adjustment $(B=-2.45$, $p<0.001)$. AHI was associated with percentage of PAR stained nuclei following adjustment $(B=13.67$, $p=0.025)$. DFU prevalence was greater in patients with OSA ( $7.1 \%$ vs. $28.1 \%$ vs. $26.2 \%$ for patients with no OSA, mild OSA and moderate to severe OSA respectively, $\mathrm{p}=0.001$ ). Following adjustment, OSA was associated with DFU (OR 3.34, 95\%Cl 1.19-9.38, p=0.022).

Conclusions: OSA is associated with lower IENFD, PARP activation and DFU in patients with Type 2 diabetes. Our finding suggest that OSA is associated with small fiber neuropathy. PARP activation is a potential mechanisms linking OSA to DPN and endothelial dysfunction in patients with Type 2 diabetes. Whether OSA treatment will have a favorable impact on these parameters and DFU requires interventional studies.

Key words: foot ulceration, neuropathy, sleep apnoea, type 2 diabetes, intra epidermal nerve fibre density, PARP 


\section{Introduction}

Obstructive sleep apnoea (OSA) is very common in patients with Type 2 diabetes and is characterized by recurrent episodes of upper airway obstruction associated with recurrent oxygen desaturations, cyclical changes in blood pressure (BP), heart rate and intrathoracic pressure, as well as recurrent arousals from sleep and changes in sleep architecture (Tahrani et al., 2013b).

We have previously shown that OSA was associated with foot insensitivity (based on 10g monofilament test) and diabetic peripheral neuropathy (DPN)(based on the Michigan Neuropathy Screening Instrument MNSI) (Tahrani et al., 2012a). These assessments are largely based on large fiber neuropathy; while the relationship between OSA and small fiber neuropathy is unknown. Intra epidermal nerve fiber density (IENFD) is considered an accurate and reproducible measure of early diabetic neuropathy and the "gold standard" for small fiber neuropathy (England et al., 2009; Lauria et al., 2010;Malik et al., 2011). Hence we hypothesized that OSA was associated with lower IENFD in patients with Type 2 diabetes.

We have also shown previously that OSA was associated with impaired cutaneous microvascular function (assessed by Laser Speckle Contrast Imaging technique) and impaired responses to nitric oxide(Tahrani et al., 2012a). OSA was associated with increased nitrosative stress and oxidative stress in patients with Type 2 diabetes which might have contributed to the associations between OSA and impaired microvascular function and DPN(Tahrani et al., 2012a). Hyperglycemia-induced nitrosative and oxidative stress play an important role in the development of vascular disease in patients with diabetes, via the activation of Poly ADP ribose polymerase (PARP), the inhibition of Glyceraldehyde 3-phosphate dehydrogenase (GAPDH) and the activation of several pathways (such as the polyol pathway and advanced-glycation end product (AGE)) resulting in inflammation, endothelial dysfunction and microvascular disease(Brownlee, 2005;Tahrani et al., 2010). Hence, we hypothesized that OSA is associated with PARP activation in patients with Type 2 diabetes which can mediate the relationship between OSA and nitrosative stress and endothelial dysfunction, which we described previously (Tahrani et al., 2012a).

PARP activation and oxidative stress have also been shown to play an important role in the pathogenesis of diabetic foot ulceration (DFU) (Tahrani et al., 2012b). Based on the association between OSA and DPN, abnormal 10g monofilament, oxidative and nitrosative stress and possibly PARP activation, we hypothesized that OSA is associated with DFU in patients with Type 2 diabetes.

Hence the primary aim of this study is to examine the relationship between OSA and IENFD and PARP activation in the skin of patients with Type 2 diabetes. A secondary aim is to explore whether there is a link between OSA and DFU in this study population. 


\section{Methods:}

We conducted an observational cross-sectional study in adults with Type 2 diabetes, the results of which have been published previously (Tahrani et al., 2012a). Patients with respiratory disease (including pre-diagnosed OSA), end-stage renal disease or non-diabetic neuropathy $(<1 \%)$ were excluded. Patients were recruited opportunistically from the out-patient diabetes departments of a UK hospital. Patients were approached in the waiting area before they have seen the clinicians and without any prior knowledge of their medical condition. We avoided any reference to snoring during the recruitment process. Consent was obtained and ethnicity self-determined in accordance with the UK decennial census by the study participants. The project was approved by the Warwickshire Research Ethics Committee (REC number 08/H1211/145) and all patients were consented accordingly.

DPN was assessed using the Michigan Neuropathy Screening Instrument (MNSI), a validated, 2component tool designed to facilitate the diagnosis of DPN, which has been widely used (1988;1999;Boyraz and Saracoglu, 2010;Feldman et al., 1994a;Martin et al., 2006;Pambianco et al., 2011).The questionnaire component (MNSIq) comprises 15 questions seeking to characterize sensory disturbance, but also peripheral vascular disease and general asthenia (Feldman et al., 1994b). The examination component (MNSle) comprises a limited foot inspection to identify deformity, skin abnormalities and ulceration, coupled with an assessment of vibratory perception at the great toe (measured using a $128 \mathrm{~Hz}$ tuning fork) and ankle tendon reflexes. DPN was diagnosed if the MNSI examination score was $>2$ and/or MNSI questionnaire score was $\geq 7$ (Martin et al., 2006;Pambianco et al., 2011). Foot insensitivity to a 10-g monofilament (applied to 10 foot locations) was defined as < eight correct responses (Pambianco et al., 2011).

OSA was assessed by a single overnight home-based cardio-respiratory sleep study using a portable multi-channel device (Alice PDX, Philips Respironics) and scored in accordance with the American Academy of Sleep Medicine guidelines (Iber C et al., 2007). An apnoea hypopnea index (AHI) $\geq 5$ events/hour was consistent with the diagnosis of OSA (Epstein et al., 2009). Sleep studies with $<4$ hours of adequate recordings were repeated and excluded if the quality remained poor. All sleep studies were scored independently by two researchers.

All patients of the original study were approached to provide skin samples to assess IENFD and \%PAR stained nuclei, and skin biopsies were performed on patients who consented. Skin biopsies were obtained from the mid-thigh level under local anesthetic and using aseptic techniques. 
IENFD was assessed as we described previously (Tahrani et al., 2012b) and recommended by others (Lauria et al., 2010). Four randomly selected $50 \mu \mathrm{m}$ sections were immunohistochemically stained using a free floating protocol with rabbit anti-human polyclonal PGP9.5 antibody (Biogenesis 1:1200). Individual ENFs were counted as they passed through the basement membrane. Negative controls comprised replacing the PG 9.5 antibody with rabbit immunoglobulin. Epidermal nerve counts were expressed as the number of fibers per millimeter of epidermis.

PARP activity was measured using immunohistochemical method that quantifies the accumulation of PAR, the product of the PARP activation (Obrosova et al., 2004; Szabó et al., 2002). PAR was assessed as we described previously (Tahrani et al., 2012b). Paraffin sections $(4 \mu \mathrm{m})$ were dewaxed and endogenous peroxidases removed and blocked. After antigen retrieval, mouse monoclonal anti PAR antibody (1:400) (Enzo Life Sciences, Exeter, UK) was applied and then washed. Sections were incubated with ImPRESS universal reagent (Vector Laboratories). Immunoreactivity was revealed using diaminobenzidine (Vector Laboratories) and sections counterstained with haematoxylin. The number of stained and unstained endothelial nuclei were counted in ten fields from each section, using 400X magnification resulting in 300-400 nuclei being assessed. The results are expressed as \% PAR stained nuclei (Szabó et al., 2002).

Foot ulceration was assessed in the whole of the original study population (i.e. not only those who consented for skin biopsies) during foot exam, and based on question 8 of the MNSIq "Have you ever had an open sore on your foot?"

All Laboratory, sleep and clinical assessments were blinded.

Data analysis was performed using SPSS 22.0 software (SPSS Inc, Chicago, USA). Data are presented as mean (SD). Differences between independent groups were assessed by analysis of variance (ANOVA). Categorical variables were compared using the Chi-squared test or Fisher's exact test. Correlations between continuous variables were performed using the Pearson or Spearman tests. Multiple linear regression (forced entry method) was used to assess independent associations of continuous outcomes and independent variables (for example the relationship between IENFD and OSA and AHI). Non-normally distributed variables were log transformed when used in the linear regression models ( $\mathrm{AHI}$ was transformed to $\log (\mathrm{AHI}+1), 1$ was added as some $\mathrm{AHI}$ values were 0$)$. Dummy variables were used in linear regression models if the independent variable was categorical with more than 2 categories (such as OSA status: no OSA, mild OSA and moderate to severe OSA). In multiple linear regression, residual distribution was examined and multicollinearity was assessed using correlations, the tolerance, variance inflation factor (VIF) and condition index. To assess 
whether OSA status or OSA severity are associated with DFU, multiple logistic regression (forced entry method) was used where DFU was the dependent variable and OSA and other variables were the independent variables. Variables included in the regression models were based on known outcome-related risk factors. A p value $<0.05$ was considered significant.

\section{Results}

Data regarding DFU was available in the whole population of the original study ( $n=234)$, of which 52 patients agreed to have skin biopsies (Figure 1). PAR staining was available for all 52 patients while IENFD results were available for 48 patients. Of the 52 patients who had skin biopsies, the prevalence of OSA was 83\% (43/52), with $24(46 \%)$ mild OSA and 19 (37\% ) moderate to severe OSA. Of the original study population the prevalence of no OSA, mild OSA and moderate-severe OSA was $36 \%(83 / 234), 39 \%(90 / 234)$ and $26 \%(61 / 234)$.

The skin biopsy study population characteristics in accordance with OSA severity are summarized in Table 1. There were no statistically significant difference between patients with and without OSA with regards to important characteristics including age, diabetes duration, lipids profile, $\mathrm{HbA1C}$, blood pressure (BP) and body mass index (BMI). There was a non-significant stepwise reduction in estimated glomerular filtration rate (eGFR) between patients with no OSA and those with mild and moderate to severe OSA as reported previously (Tahrani et al., 2013a). Patients with OSA had higher MNSle scores indicating more DPN in patients with OSA as previously reported (Tahrani et al., 2012a). There were more men and more White Europeans in patients with OSA, but these differences were not statistically significant. There were no differences in alcohol intake or smoking between groups. Insulin prescription was similar across groups and a high proportion of all groups were prescribed BP lowering and lipid lowering treatments.

\section{OSA and IENFD}

Results of IENFD were available in 48 patients (4 patients did not have enough tissue left to perform PAR immunostaining but not IENFD) , 9 patients with no OSA, 22 with mild OSA and 17 with moderate to severe OSA.

In ANOVA, there was a significant reduction in IENFD across groups ( $12.75 \pm 1.93$ vs. $10.55 \pm 1.62$ vs. $9.42 \pm 1.16$ fibers $/ \mathrm{mm}$ of epidermis for no OSA, mild OSA and moderate to severe OSA respectively, $p<0.001)$ (Figure 2). IENFD correlated negatively with the AHI $(r=-0.51, p<0.001)$ and ODI $(r=-0.33$, $\mathrm{p}=0.02$ ). There were no associations between IENFD and time spent with oxygen saturation $<90 \%$ $(r=0.08, p=0.57)$ or nadir nocturnal oxygen saturation $(r=0.02, p=0.9)$. 
Using multiple linear regression, where IENFD was the dependent variable and OSA, age, gender, ethnicity, diabetes duration, $\mathrm{HbA} 1 \mathrm{c}$ and $\mathrm{BMI}$ were the independent variables $\left(\mathrm{R}^{2}=0.43\right)$; mild OSA $(B=-2.19, p=0.002)$ and moderate to severe OSA $(B=-3.45, p<0.001)$ were associated with IENFD. Replacing OSA with log $(A H I+1)$ in the model showed that IENFD was associated with log $(A H I+1)$ $\left(R^{2}=0.37\right)(B=-2.45, p<0.001)$. The regression coefficient suggests that lower $A H I$ values were associated with higher IENFD. Similar results were found when log $(O D I+1)$ was used in the model instead of $\log (A H I+1)\left(R^{2}=0.29\right)(B=-2.10, p=0.004)$.

\section{OSA and PAR}

There was a statistically non-significant trend of increasing \%PAR stained nuclei with worsening OSA (55.17 \pm 20.91 vs. $58.89 \pm 17.24$ vs. $66.84 \pm 15.73$ for patients with no OSA, mild OSA and moderate to severe OSA respectively, $p=0.18)$ (Figure 3$)$. Log $(A H I+1)$ correlated with \%PAR stained nuclei $(r=0.29$, $\mathrm{p}=0.039)$ but there was no significant correlation between $\log (\mathrm{ODI}+1)$ and $\% \mathrm{PAR}$ stained nuclei $(r=0.23, p=0.097)$.

Using the same multiple linear regression model described above but replacing IENFD with \%PAR stained nuclei as the dependent variable $\left(R^{2}=0.20\right)$ showed no relationship between \%PAR stained nuclei and mild OSA ( $B=5.86, p=0.4$,) or moderate to severe OSA ( $B=14.33, p=0.068)$. However, there was an association between $\log (A H I+1)$ and \%PAR stained nuclei when log $(A H I+1)$ was used in the model instead of OSA ( $B=13.67, p=0.025)$. The regression coefficient suggests that higher $A H I$ was associated with higher \%PAR stained nuclei.

\section{OSA and DFU}

The baseline characteristics of the original study $(n=234)$ in relation to OSA status are summarized in Table 2 and were published previously(Tahrani et al., 2012a). Patients with OSA were older, heavier and had longer diabetes duration and higher prevalence of peripheral vascular disease (PVD, based on the patients electronic records). OSA was also more common in men and in White Europeans. OSA patients were prescribed more insulin and anti-hypertensive medication compared to those without OSA.

The prevalence of DFU on clinical examination was $3.4 \%(n=8)$. There was a stepwise increase in the prevalence of DFU in patients with no OSA vs. mild OSA vs. moderate to severe OSA (0\% (0) vs. $2.2 \%$ (2) vs. $10 \%(6), p=0.004)$. Due to the small number of patients with DFU on clinical examination, we could not perform logistic regression to assess independent associations.

Based on question 8 of the MNSIq, 47/234 (20.1\%) patients had past history of DFU. The prevalence of a past history of DFU was significantly greater in patients with OSA (7.1\% (6/84) vs. $28.1 \%(25 / 89)$ 
vs. $26.2 \%$ (16/61) for patients with no OSA, mild OSA and moderate to severe OSA respectively, $\left.X^{2}=13.75, p=0.001\right)$.

Using the presence of DFU past history (based on the MNSIq) as the dependent variable and OSA, age, gender, ethnicity, diabetes duration, Presence of PVD, BMI, HbA1c, and insulin treatment (Nagelkerke $\mathrm{R}^{2}=0.25$ ) showed that OSA was associated with past history of DFU (OR $3.34,95 \% \mathrm{Cl}$ 1.19-9.38, $p=0.022$ ). Using the same model but replacing OSA (binary yes/no category) with OSA severity showed that mild OSA was associated with past history of DFU (OR 3.69, 95\%Cl 1.28-10.63, $\mathrm{p}=0.016$ ) and there was a statistically non-significant association with moderate to severe OSA (OR $2.88,95 \% \mathrm{Cl} 0.87-9.54, \mathrm{p}=0.084)$.

\section{Conclusions}

We have shown that OSA and AHI are associated with lower IENFD, AHI is associated with PARP activation and OSA is associated with DFU in patients with Type 2 diabetes. To the best of our knowledge, this is the first report to describe the relationships between OSA and IENFD, PARP activation and DFU in patients with Type 2 diabetes.

IENFD has been shown to have high sensitivity and specificity to diagnose DPN, particularly small fiber neuropathy (England et al., 2009;Lauria et al., 2010;Malik et al., 2011). Intra- and interobserver variability for the assessment of IENFD demonstrates good agreement (Malik et al., 2011). IENFD was found to correlate inversely with thermal thresholds and pain severity(Malik et al., 2011). The lower IENFD in patients with OSA and Type 2 diabetes vs. patients with Type 2 diabetes only suggest that OSA is associated with small fiber neuropathy in patients with Type 2 diabetes which complements our previous finding that OSA was associated with clinically evident DPN and foot insensitivity suggesting that OSA in patients with Type 2 diabetes is associated with both small and large fibers neuropathy. Even mild OSA was independently associated with lower IENFD, which might reflect the relatively long diabetes duration in these patients that could amplify the impact of mild OSA in vulnerable tissues. Thus, assessing patients with shorter diabetes duration might yield different results. This finding was also similar to our previous study showing that mild OSA was independently associated with clinically evident DPN(Tahrani et al., 2012a). Obesity also is an established risk factor for DPN and SDB (Tahrani et al., 2013b;Tesfaye et al., 2005); in addition IENFD has been shown to correlate negatively with BMI (Smith and Singleton, 2013). However, in this study there were no significant differences in BMI between groups and the associations between IENFD and SDB remained significant despite adjustment for BMI in the multivariable analysis. 
PARP activation, secondary to oxidative stress-induced DNA damage, has been shown to play an important role in the pathogenesis of DPN and endothelial dysfunction in patients with diabetes (Obrosova et al., 2004;Szabó et al., 2002). In our study, PARP activation was independently associated with higher AHI; this is likely to be due to DNA damage secondary to oxidative stress in patients with OSA and type 2 diabetes. We have shown previously that OSA in patients with type 2 diabetes was associated with increased oxidative and nitrosative stress (Tahrani et al., 2012a). In addition, PARP activation offers another possible mechanism to explain the cross-sectional and longitudinal associations observed between OSA and microvascular complications and endothelial dysfunction observed in patients with type 2 diabetes (Altaf et al., 2015; Tahrani et al., 2012a;Tahrani et al., 2013a;Tahrani et al., 2013b). PARP inhibition has been shown to alleviate oxidative stress and reverse DPN and neuropathy in diabetic rodents (Drel et al., 2010;Drel et al., 2011; Lupachyk et al., 2011). Intermittent hypoxia has been shown to result in oxidative stress and PARP activation in invitro non-diabetic rodents (Chiu et al., 2012).

We also found that OSA is independently associated with DFU in patients with Type 2 diabetes. This association was independent of important risk factors such as PVD, BMI, age, diabetes duration and HbA1c. Considering that OSA is associated with DPN, endothelial dysfunction, oxidative stress, nitrosative stress, lower IENFD and PARP activation, this association between OSA and DFU may not be surprising. A recent in-vitro study showed that the serum from patients with OSA and the metabolic syndrome resulted in impaired endothelial wound healing compared to healthy controls (Briançon-Marjollet et al., 2014). However, a small study of 25 patients with OSA showed no evidence of impaired wound healing following partial foot amputation (Andrews et al., ). A recently published case series $(n=3)$ suggested that CPAP treatment aided DFU healing in 2 patients with undiagnosed OSA and DFU (Vas et al., 2015). The impact of OSA on DFU and wound healing in patients with type 2 diabetes requires further prospective and interventional studies.

This study has several limitations, most importantly its cross-sectional design where causality cannot be proven. Although several reasons might explain how OSA might result in lower IENFD, PARP activation and DFU, it is plausible for neuropathy to result or worsen OSA by its impact on upper airway function and the ventilatory drive. Hence, it is possible that the relationship could be bidirectional. The rather small sample size is another limiting factor but not many patients were willing to have skin biopsy which is a mini-invasive procedure, but 52 patients is comparable (and even more) than many other studies of skin biopsies in patients with Type 2 diabetes.

Our study also has strengths. The population of the study were well characterized and this is the first report to examine the relationship between OSA and IENFD, PARP and DFU in patients with type 2 
diabetes. This is also the first report to show a relationship between OSA and IENFD and PARP activation in humans.

In summary, OSA was associated with lower IENFD, PARP activation and DFU in patients with Type 2 diabetes. PARP activation could be one of the mechanisms relating OSA to microvascular dysfunction and complications in patients with Type 2 diabetes. Whether PARP inhibition would be beneficial requires further studies. The impact of OSA on IENFD, PARP and DFU requires further prospective studies. Whether continuous positive airway pressure treatment can improve IENFD, inhibit PARP and/or reduce the incidence/improve the healing of DFU requires interventional studies.

\section{Authors contributions:}

QAA: researched data, wrote and reviewed manuscript

AA: researched data, reviewed the manuscript

MKP: researched data, reviewed the manuscript

NTR: Reviewed paper and reviewed statistical analysis

AAT: hypothesis, obtained funding, researched data, laboratory work, wrote and reviewed manuscript

Acknowledgment: 
We thank Dr. Sharon Hughes for her help with the immunostaining. A. A. T. is a clinician scientist supported by the National Institute for Health Research in the UK. The views expressed in this publication are those of the author(s) and not necessarily those of the National Health Service, the National Institute for Health Research or the Department of Health. AAT is the guarantor of the study. 


\section{REFERENCES}

1. 1988. Factors in development of diabetic neuropathy. Baseline analysis of neuropathy in feasibility phase of Diabetes Control and Complications Trial (DCCT). The DCCT Research Group. Diabetes 37, 476-481.

2. 1999. Epidemiology of Diabetes Interventions and Complications (EDIC). Design, implementation, and preliminary results of a long-term follow-up of the Diabetes Control and Complications Trial cohort. Diabetes Care 22, 99-111.

3. Altaf, Q.A., Barnett, A.H., Tahrani, A.A., 2015. Novel therapeutics for type 2 diabetes: insulin resistance. Diabetes Obes. Metab 17, 319-334.

4. Andrews, K.L., Dib, M., Shives, T.C., Hoskin, T.L., Liedl, D.A., Boon, A.J., The effect of obstructive sleep apnea on amputation site healing. Journal of Vascular Nursing 30, 61-63.

5. Boyraz, O., Saracoglu, M., 2010. The effect of obesity on the assessment of diabetic peripheral neuropathy: A comparison of Michigan patient version test and Michigan physical assessment. Diabetes research and clinical practice 90, 256-260.

6. Briançon-Marjollet, A., Henri, M., Pépin, J.L., Lemarié, E., Lévy, P., Tamisier, R., 2014. Altered in vitro Endothelial Repair and Monocyte Migration in Obstructive Sleep Apnea: Implication of VEGF and CRP. Sleep 37, 1825.

7. Brownlee, M., 2005. The Pathobiology of Diabetic Complications: A Unifying Mechanism. Diabetes 54, 1615-1625.

8. Chiu, S.C., Huang, S.Y., Tsai, Y.C., Chen, S.P., Pang, C.Y., Lien, C.F., Lin, Y.J., Yang, K.T., 2012. Poly (ADP-ribose) polymerase plays an important role in intermittent hypoxia-induced cell death in rat cerebellar granule cells. Journal of Biomedical Science 19, 29.

9. Drel, V.R., Lupachyk, S., Shevalye, H., Vareniuk, I., Xu, W., Zhang, J., Delamere, N.A., Shahidullah, M., Slusher, B., Obrosova, I.G., 2010. New Therapeutic and Biomarker Discovery for Peripheral Diabetic Neuropathy: PARP Inhibitor, Nitrotyrosine, and Tumor Necrosis Factor- $\alpha$. Endocrinology 151, 2547-2555.

10. Drel, V.R., Pacher, P., Stavniichuk, R., Xu, W., Zhang, J., Kuchmerovska, T.M., Slusher, B., Obrosova, I.G., 2011. Poly (ADP-ribose) polymerase inhibition counteracts renal hypertrophy and multiple manifestations of peripheral neuropathy in diabetic Akita mice. International journal of molecular medicine 28, 629-635.

11. England, J.D., Gronseth, G.S., Franklin, G., Carter, G.T., Kinsella, L.J., Cohen, J.A., Asbury, A.K., Szigeti, K., Lupski, J.R., Latov, N., Lewis, R.A., Low, P.A., Fisher, M.A., Herrmann, D.N., Howard, J.F., Lauria, G., Miller, R.G., Polydefkis, M., Sumner, A.J., 2009. Practice Parameter: Evaluation of distal symmetric polyneuropathy: Role of autonomic testing, nerve biopsy, and skin biopsy (an evidence-based review). Neurology 72, 177-184.

12. Epstein, L.J., Kristo, D., Strollo, P.J., Jr., Friedman, N., Malhotra, A., Patil, S.P., Ramar, K., Rogers, R., Schwab, R.J., Weaver, E.M., Weinstein, M.D., 2009. Clinical guideline for the evaluation, management and long-term care of obstructive sleep apnea in adults. J Clin Sleep Med 5, 263-276. 
13. Feldman, E.L., Stevens, M.J., Thomas, P.K., Brown, M.B., Canal, N., Greene, D.A., 1994a. A practical two-step quantitative clinical and electrophysiological assessment for the diagnosis and staging of diabetic neuropathy. Diabetes Care 17, 1281-1289.

14. Feldman, E.L., Stevens, M.J., Thomas, P.K., Brown, M.B., Canal, N., Greene, D.A., 1994b. A practical two-step quantitative clinical and electrophysiological assessment for the diagnosis and staging of diabetic neuropathy. Diabetes Care 17, 1281-1289.

15. Iber C, Ancoli-Israel S, Chesson A, Quan S, 2007. The AASM manual for the scoring of sleep and associated events: rules, terminology and technical specifications. American Academy of Sleep Medicine, Westchester: IL.

16. Lauria, G., Hsieh, S.T., Johansson, O., Kennedy, W.R., Leger, J.M., Mellgren, S.I., Nolano, M., Merkies, I.S.J., Polydefkis, M., Smith, A.G., Sommer, C., Valls-Sol+ ${ }^{\circledR}$, J., 2010. European Federation of Neurological Societies/Peripheral Nerve Society Guideline on the use of skin biopsy in the diagnosis of small fiber neuropathy. Report of a joint task force of the European Fe-deration of Neurological Societies and the Peripheral Nerve Society. European Journal of Neurology 17, 903-e49.

17. Lupachyk, S., Shevalye, H., Maksimchyk, Y., Drel, V.R., Obrosova, I.G., 2011. PARP inhibition alleviates diabetes-induced systemic oxidative stress and neural tissue 4-hydroxynonenal adduct accumulation: Correlation with peripheral nerve function. Free Radical Biology and Medicine 50, 1400-1409.

18. Malik, R.A., Veves, A., Tesfaye, S., Smith, G., Cameron, N., Zochodne, D., Lauria, G., on behalf of The Toronto Consensus Panel on Diabetic Neuropathy, 2011. Small fibre neuropathy: role in the diagnosis of diabetic sensorimotor polyneuropathy. Diabetes/Metabolism Research and Reviews 27, 678-684.

19. Martin, C.L., Albers, J., Herman, W.H., Cleary, P., Waberski, B., Greene, D.A., Stevens, M.J., Feldman, E.L., 2006. Neuropathy Among the Diabetes Control and Complications Trial Cohort 8 Years After Trial Completion. Diabetes Care 29, 340-344.

20. Obrosova, I.G., Li, F., Abatan, O.I., Forsell, M.A., Komjáti, K., Pacher, P., Szabó, C., Stevens, M.J., 2004. Role of Poly(ADP-Ribose) Polymerase Activation in Diabetic Neuropathy. Diabetes 53, 711-720.

21. Pambianco, G., Costacou, T., Strotmeyer, E., Orchard, T.J., 2011. The assessment of clinical distal symmetric polyneuropathy in type 1 diabetes: A comparison of methodologies from the Pittsburgh Epidemiology of Diabetes Complications Cohort. Diabetes research and clinical practice.

22. Smith, A.G., Singleton, J.R., 2013. Obesity and hyperlipidemia are risk factors for early diabetic neuropathy. Journal of Diabetes and its Complications 27, 436-442.

23. Szabó, C., Zanchi, A., Komjáti, K., Pacher, P.+., Krolewski, A.S., Quist, W.C., LoGerfo, F.W., Horton, E.S., Veves, A., 2002. Poly(ADP-Ribose) Polymerase Is Activated in Subjects at Risk of Developing Type 2 Diabetes and Is Associated With Impaired Vascular Reactivity. circulation 106, 2680-2686.

24. Tahrani, A.A., Ali, A., Raymond, N.T., Begum, S., Dubb, K., Mughal, S., Jose, B., Piya, M., Barnett, A.H., Stevens, M.J., 2012a. Obstructive Sleep Apnea and Diabetic Neuropathy: a Novel Association in Patients with Type 2 Diabetes. Am J Respir Crit Care Med 186, 434-441. 
25. Tahrani, A.A., Askwith, T., Stevens, M.J., 2010. Emerging drugs for diabetic neuropathy. Expert. Opin. Emerg. Drugs 15, 661-683.

26. Tahrani, A.A., Zeng, W., Shakher, J., Piya, M.K., Hughes, S., Dubb, K., Stevens, M.J., 2012b. Cutaneous structural and biochemical correlates of foot complications in high-risk diabetes. Diabetes Care 35, 1913-1918.

27. Tahrani, A.A., Ali, A., Raymond, N.T., Begum, S., Dubb, K., Altaf, Q.A., Piya, M.K., Barnett, A.H., Stevens, M.J., 2013a. Obstructive Sleep Apnea and Diabetic Nephropathy: A cohort study. Diabetes Care 36, 3718-3725.

28. Tahrani, A.A., Ali, A., Stevens, M.J., 2013b. Obstructive sleep apnoea and diabetes: an update. Current Opinion in Pulmonary Medicine 19, 631-638.

29. Tesfaye, S., Chaturvedi, N., Eaton, S.E.M., Ward, J.D., Manes, C., lonescu-Tirgoviste, C., Witte, D.R., Fuller, J.H., 2005. Vascular Risk Factors and Diabetic Neuropathy. New England Journal of Medicine 352, 341-350.

30. Vas, P.R.J., Ahluwalia, R., Manas, A.B., Manu, C.A., Kavarthapu, V., Edmonds, M.E., 2015. Undiagnosed severe sleep apnoea and diabetic foot ulceration $\Gamma$ Çô a case series based hypothesis: a hitherto under emphasized factor in failure to heal. Diabetic Medicine n/a. 
Table 1: The characteristics of patients who had skin biopsies in relation to OSA status. BP: blood pressure; BMI: body mass index; PVD: peripheral vascular disease; eGFR: estimated glomerular filtration rate, EDS: excessive daytime sleepiness (i.e. Epworth sleepiness score $\geq 11$ ).

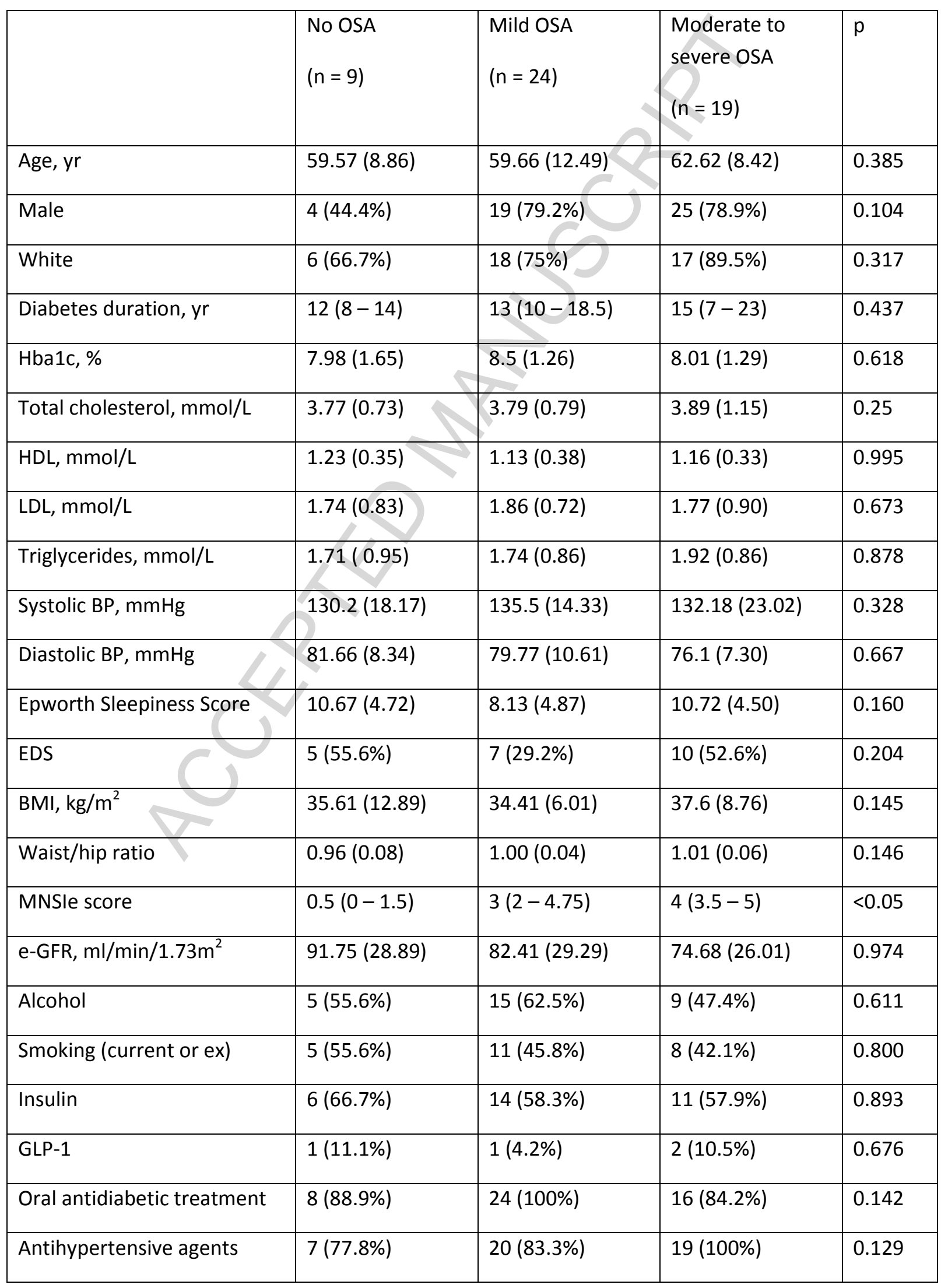




\begin{tabular}{|l|l|l|l|l|}
\hline Lipid lowering treatment & $9(100 \%)$ & $20(83.3 \%)$ & $19(100 \%)$ & 0.080 \\
\hline PVD & $0(0.0 \%)$ & $1(4.2 \%)$ & $3(15.8 \%)$ & 0.232 \\
\hline
\end{tabular}

Table 2: Participant characteristics in relation to SDB status. Data presented as median (IQR) or mean (SD). Categorical variables presented as number (\% of SDB status).GFR: Glomerular Filtration Rate, TIA: Transient Ischaemic Attack, PVD: Peripheral Vascular Disease, EDS: Excessive Daytime Sleepiess. Analysis performed using the Chi-square test for categorical variables, the independent $t$ test for normally distributed variables and the Mann-Whitney $U$ test for non-normally distributed variables. Adapted with permission from (2).

\begin{tabular}{|l|l|l|l|}
\hline & OSA- $(\mathrm{n}=83)$ & OSA $(\mathrm{n}=151)$ & P value \\
\hline Male & $34(41 \%)$ & $101(67 \%)$ & $<0.001$ \\
\hline Caucasians & $32(39 \%)$ & $97(64 \%)$ & $<0.001$ \\
\hline Age (years) & $54.7(11.9)$ & $58.5(11.3)$ & 0.02 \\
\hline Diabetes Duration (years) & $9(5-15)$ & $11(7-17)$ & 0.02 \\
\hline Body Mass Index (kg/m $\left.{ }^{2}\right)$ & $30.2(27.3-35.0)$ & $34.4(30.9-39.5)$ & $<0.001$ \\
\hline Waist circumference (cm) & $105.5(96.0-115.0)$ & $116.0(107.5-125.5)$ & $<0.001$ \\
\hline Hip (cm) & $106.0(98.0-117.0)$ & $114.0(105.0-125.0)$ & $<0.001$ \\
\hline Waist hip ratio & $0.97(0.93-1.02)$ & $1.01(0.96-1.05)$ & 0.002 \\
\hline Neck circumference (cm) & $38.0(36.5-41.3)$ & $43.0(39.0-46.0)$ & $<0.001$ \\
\hline Height (cm) & $163.5(8.3)$ & $167.8(10.0)$ & 0.001 \\
\hline Systolic blood pressure (mmHg) & $125.5(115.0-135.5 .0)$ & $130.0(123.5-140.0)$ & 0.002 \\
\hline Diastolic blood pressure (mmHg) & $78.50(71.0-85.00)$ & $78.00(71.00-84.50)$ & 0.88 \\
\hline HbA1c (\%) & $7.7(7.0-8.7)$ & $8.3(7.3-9.3)$ & 0.05 \\
\hline Total cholesterol (mmol/L) & $3.7(3.4-4.5)$ & $3.7(3.3-4.3)$ & 0.57 \\
\hline Triglycerides (mmol/L) & $1.5(1.0-2.1)$ & $1.8(1.3-2.5)$ & 0.02 \\
\hline HDL (mmol/L) & $1.2(0.9-1.4)$ & $82.41(26.41)$ & \\
\hline Estimated GFR (ml/min/1.73 m²) & $92.92(25.16)$ & & \\
\hline
\end{tabular}




\begin{tabular}{|l|l|l|l|}
\hline TSH & $1.6(1.0-2.2)$ & $1.7(1.2-2.4)$ & 0.32 \\
\hline Epworth sleepiness score & $5.0(2.0-12.0)$ & $8.0(4.0-13.0)$ & 0.003 \\
\hline EDS & $30.1 \%(\mathrm{n}=25)$ & $39.1 \%(\mathrm{n}=59)$ & 0.172 \\
\hline Smoking (current or ex-smoker) & $32(39 \%)$ & $62(41 \%)$ & 0.71 \\
\hline Alcohol (drinks alcohol) & $12(15 \%)$ & $12(35 \%)$ & 0.001 \\
\hline Oral anti-diabetes treatment & $81(98 \%)$ & $137(91 \%)$ & 0.05 \\
\hline Insulin & $34(41 \%)$ & $91(60 \%)$ & 0.005 \\
\hline Insulin dose (units) & $61(35-88)$ & $80(56-118)$ & 0.007 \\
\hline ACE inhibitors & $40(48 \%)$ & $69(46 \%)$ & 0.71 \\
\hline Anti-hypertensive agents & $61(74 \%)$ & $129(85 \%)$ & 0.03 \\
\hline Lipid lowering treatment & $71(86 \%)$ & $125(83 \%)$ & 0.58 \\
\hline Stroke or TIA & $60(7 \%)$ & $18(12 \%)$ & 0.28 \\
\hline Ischemic heart disease & $14(17 \%)$ & $33(22 \%)$ & 0.40 \\
\hline PVD & $1(1 \%)$ & $10(7 \%)$ & 0.001 \\
\hline Albuminuria & $20(24 \%)$ & $65(48 \%)$ & \\
\hline Sight threatening retinopathy & $17(21 \%)$ & & \\
\hline & & & \\
\hline
\end{tabular}


Figure 1: The study diagram

\section{Consented and enrolled in the study \\ and had sleep studies and other data collected $(n=266)$}

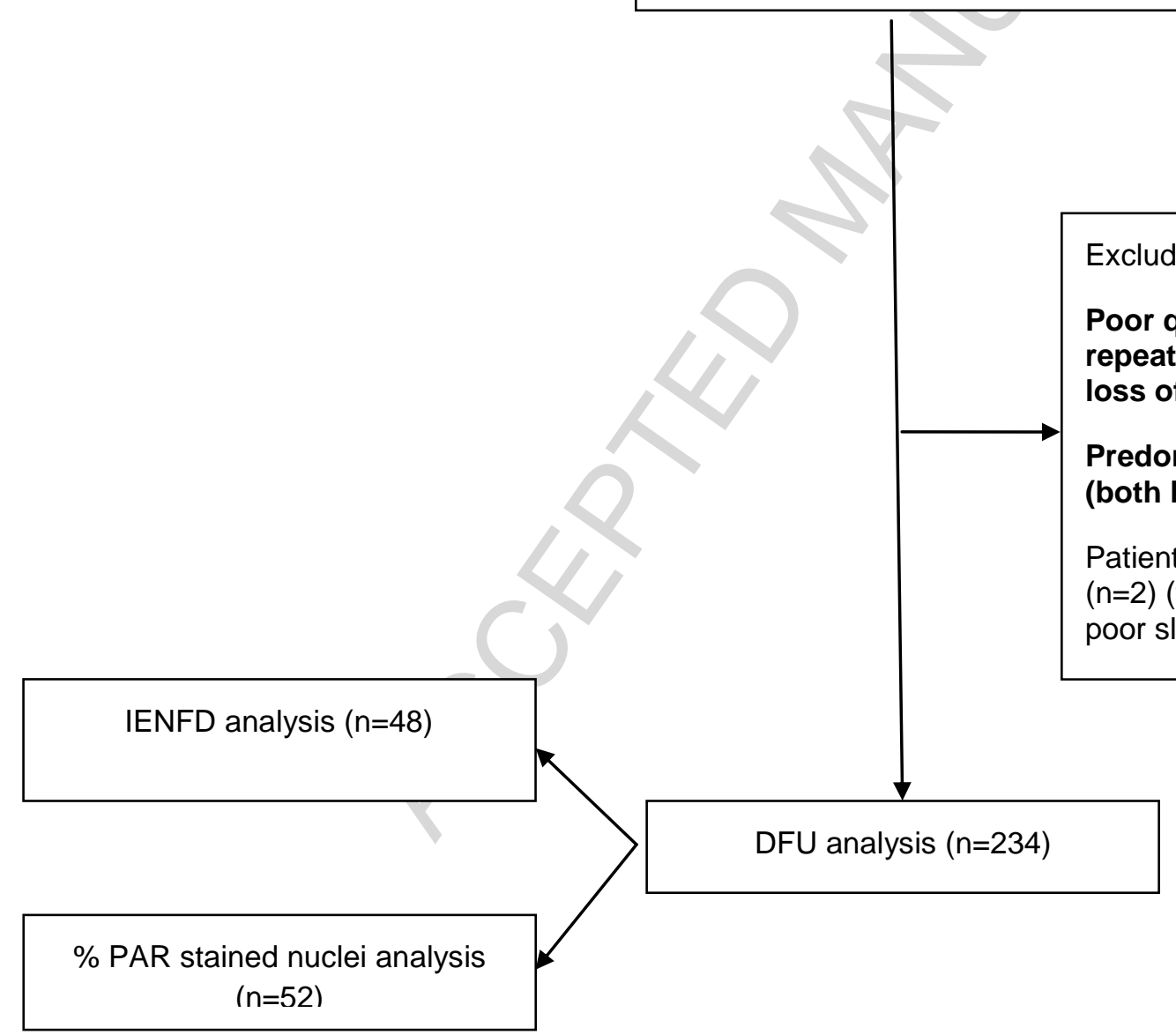


Figure 2 - The relationship between obstructive sleep apnoea and Intra epidermal nerve fibre density (IENFD).

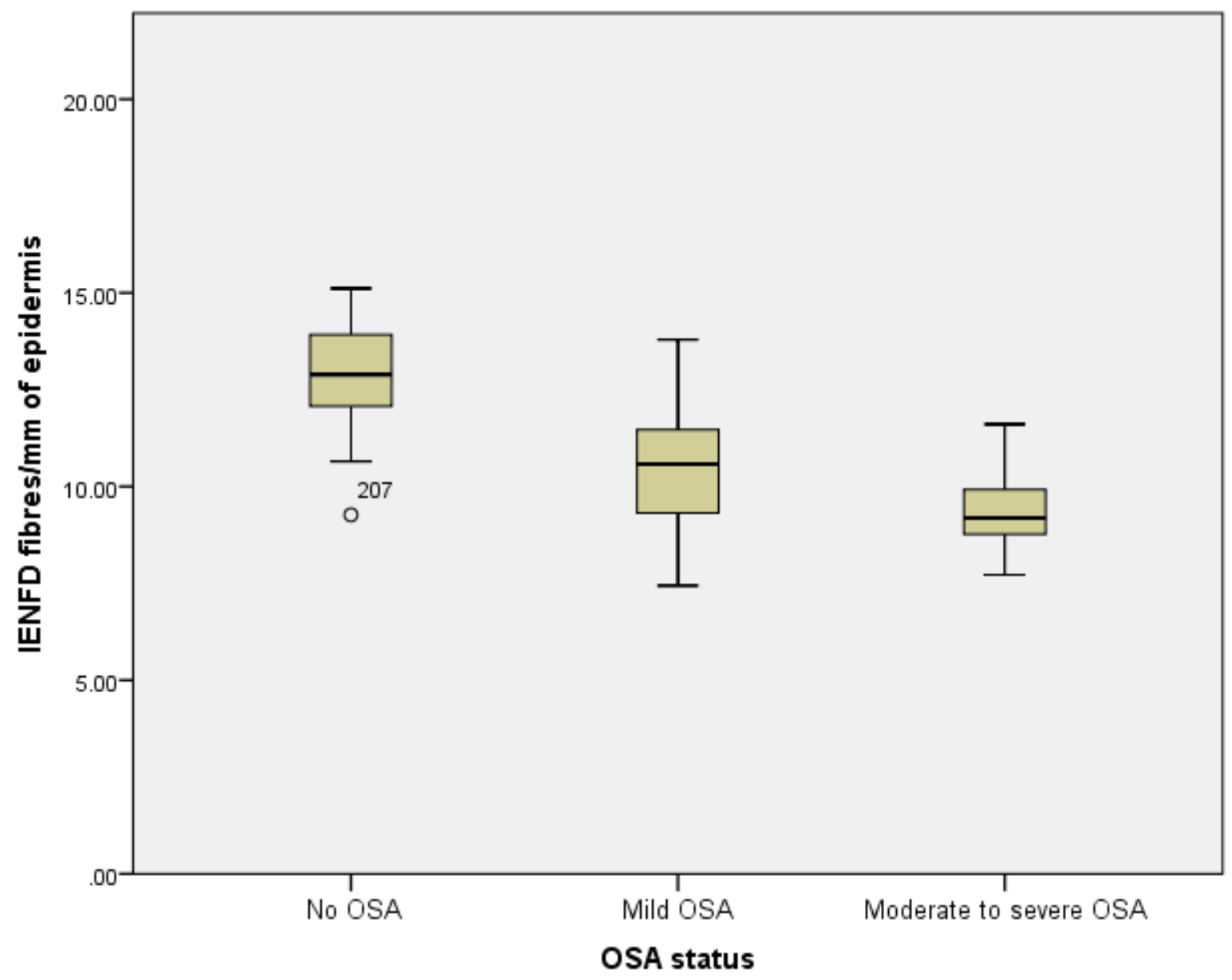


Figure 3: PAR immunostaining from A) patient with AHI of 1.6, B) patient with AHI 31.2. Black arrow: non PAR stained nuclei (purple), Red arrow: PAR stained nuclei (brown)

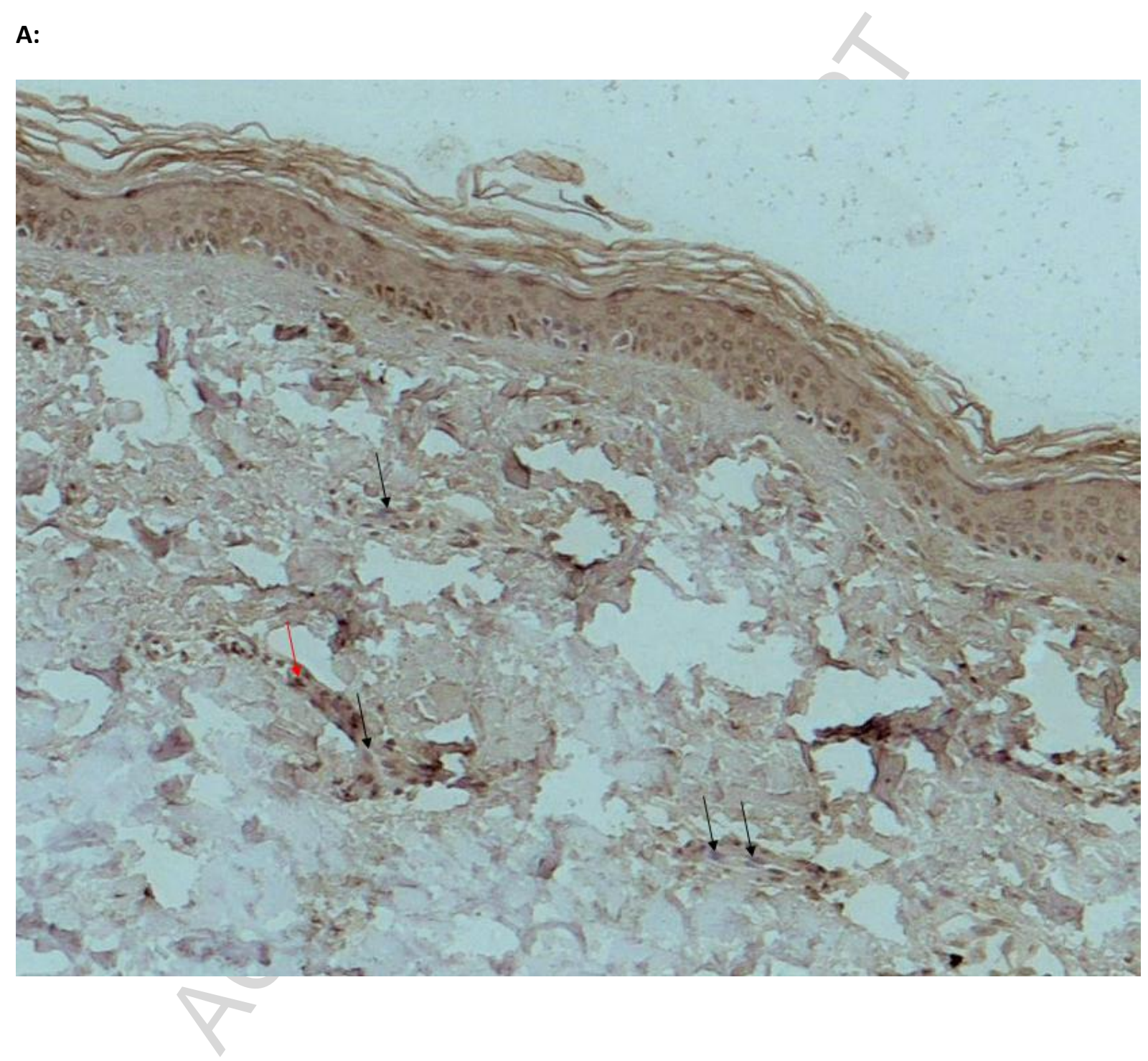


B:

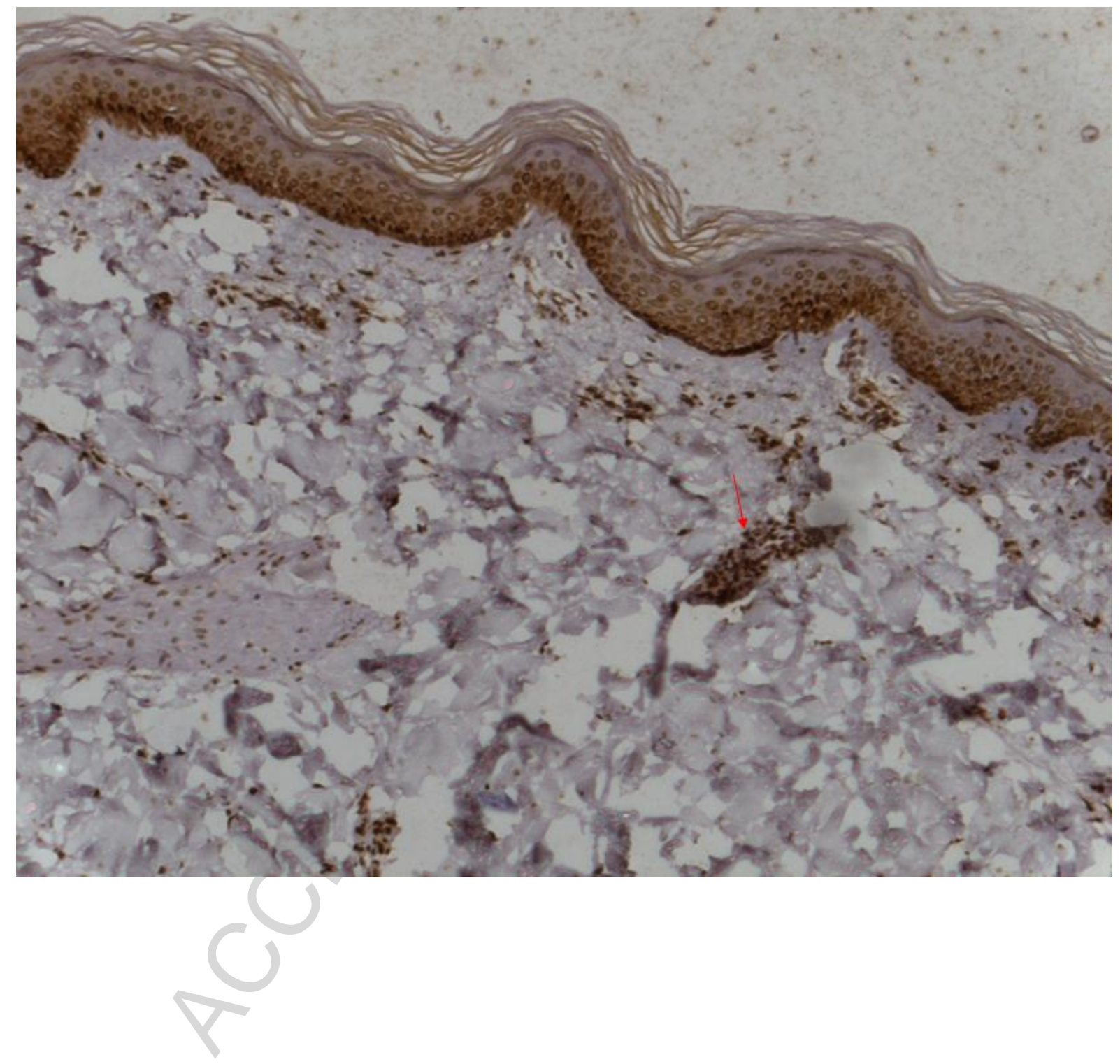




\section{Highlights}

1. OSA is associated with lower IENFD in patients with Type 2 diabetes (T2D)

2. OSA is associated with small as well as large fibre dysfunction in patients with T2D.

3. OSA is associated with PARP activation in patients with T2D

4. OSA is associated with foot ulceration in patients with T2D.

5. The impact of CPAP on IENFD and foot ulceration in T2D need to be examined. 